\title{
Logic and Fallacy
}

\section{Kuan Tsee Chee*}

Institute of Mental Health, Singapore

\section{Short Communication}

Evidence Based Medicine begins with anecdotal observation and empirical experience. Or anecdotal observation and empirical experience are the bases for evidence based medicine. To insist rhetorically on "evidence" first before initiating any new potentially beneficial treatment is to think in the box and impede innovation and progress. There would have been no discovery of the antibiotic penicillin and vaccination of chicken pox. To reiterate, evidence based derives from experience based.

Randomized Clinical Drug Trials in psychiatry are based on diagnoses according to DSM or ICD. Such diagnoses are mostly syndromes decided by "authoritative" consensus of opinions and not universal. As a matter of fact many psychiatric diagnoses are groups of heterogeneous disorders. It is recognized in clinical practice that mental disorders are multifactorial in causality and multidisciplinary in management. The overall outcomes of specific drugs used are similar in one third therapeutic efficacy or up to two thirds variable response and not excluding placebo effect. What differs is in side effect profile and perhaps specific symptom(s) amelioration.

The clinicians more often than not are guided in their prescriptions by their practical experience, knowledge and understanding of psychopathology, pathophysiology and individual psychosocial stressors of each patient besides factors such as availability, affordability and lifestyle. Such individualized management would give better results than one size fits, trial and error algorithm. Inadvertently, the so called "off label" dynamic polypharmacy creeps in. RCDT would therefore preferably be based on psychopathology and pathophysiology rather than "specific" diagnosis. Hence we do not talk of "anti-schizophrenic" or "anti-bipolar" drugs even when RCDT are based on diagnoses of schizophrenia and bipolar disorder. We prescribe instead anti- psychotics or mood stabilizers. There is already a trend to shift focus on management of specific domain of psychopathology such as functional cognitive deficit in depressive illness. It involves parameters of social function, work capacity and productivity apart from sleep, mood and libido etc.

Biomarkers are questionable when diagnoses are syndromal, non nosological, heterogeneous with shared genes and similar symptomatology. Besides the biomarkers could be "cause" or "effect". However, biomarkers are being looked into in terms of treatment response.

In general the practice of Evidence Based Medicine is controlled by FDA and other international academic or professional bodies and individual National Regulations. What is approved and available in one country may not be the same as in another. They are not binding, comprehensive and infallible. In fact they may impede discovery and progress.

\section{Psychotherapy}

Pharmacotherapy has come to a sort of "dead end" in recent years with most patented drugs becoming generic and with very limited new drugs of novel mechanisms on the horizon. It is not surprising that psychotherapy is swinging back because RCDT has omitted psychosocial factors in mental disorders which are important in understanding morbidity and management. However, there are many schools, theories and models on psychopathology and therefore variety of psychotherapy. Psychotherapy probably works through plastic rewiring of the neuro-circuitries through systematic and strategic cognitive and behavioral input while pharmacotherapy attempts to restore balance of neurotransmitters and synaptic receptors.
*Corresponding author: Kuan Tsee Chee, Institute of Mental Health, Singapore, Tel: 63892000; E-mail: kuan_tsee_chee@imh.com.sg

Received September 04, 2015; Accepted May 31, 2015; Published June 07 2015

Citation: Chee KT (2015) Logic and Fallacy. J Psychiatry 18: 300 doi 10.4172/2378-5756.1000300

Copyright: ( 2015 Chee KT. This is an open-access article distributed under the terms of the Creative Commons Attribution License, which permits unrestricted use, distribution, and reproduction in any medium, provided the original author and source are credited 